\title{
LE POISSON ET SON HABITAT : INTRODUCTION DU SYMPOSIUM.
}

\author{
Y. SOUCHON (1), C. LÉVÊQUE (2), P. GAUDIN (3)
}

(1) Cemagref, Laboratoire d'Hydroécologie Quantitative, Division Biologie des Ecosystèmes Aquatiques, 3 bis quai Chauveau, CP 220, 69336 Lyon Cedex 09, France.

(2) Orstom Direction, 213 rue La Fayette, 75480 Paris Cedex 10, France.

(3) Laboratoire d'Ecologie des Eaux Douces et des Grands Fleuves, URA CNRS 1974, Université Lyon I, 43 bd du 11 Novembre 1918, 69622 Villeurbanne Cedex, France.

\section{RÉSUMÉ}

Le symposium international «Le poisson et son habitat» a permis une rencontre entre deux communautés de scientifiques travaillant soit à l'échelle régionale, soit à l'échelle locale. Ce fut l'occasion de synthèses régionales originales et de comparaisons intercontinentales des peuplements de poisson. Beaucoup de précisions ont été produites sur l'habitat ontogénétique des poissons et des salmonidés en particulier. Les approches modélisatrices de l'habitat s'enrichissent de nouvelles options de description du compartiment physique, de nouveaux modèles d'habitat et bénéficient désormais d'un certain retour d'expérience qui profitera aux actions de préservation et de restauration des milieux aquatiques. La réalisation d'une réelle gestion intégrée de ces milieux passera par la maîtrise théorique et pratique de ces différentes échelles et par un investissement de recherche équilibré entre physique et biologie.

\section{FISH AND THEIR HABITAT : INTRODUCTION OF THE SYMPOSIUM.}

\section{SUMMARY}

The international symposium «Fish and their habitat» made it possible to meet two scientific communities working either at a regional scale or at a local scale. This meeting was an occasion for presenting original regional synthesis and intercontinental comparisons of fish assemblages. Many original results were presented on ontogenetic fish habitat, especially for salmonids. Modelling approaches of habitat are now enriched with new modes of description of the physical compartment, new habitat models, and they now profit by some experience which will be of benefit to the preservation and restoration of aquatic environments. The accomplishment of a truly integrated management of these environments will now depend on the theoretical and practical control of the different scales and on a well balanced investment of both physical and biological research.

\section{INTRODUCTION}

La reconnaissance du rôle déterminant joué par l'habitat vis-à-vis des populations de poisson n'est pas récente. LÉGER (1909) signalait déjà son importance et attribuait aux premières centrales hydroélectriques installées dans les Alpes la dégradation des populations de truite (Salmo trutta fario). Paradoxalement, il faut attendre de nombreuses années et la fin de la décennie 1970 (WATERS, 1976) pour que soient jetées les bases d'une réelle quantification des effets biologiques des modifications des régimes hydrologiques. Depuis, les progrès sont allés grandissant, les publications se sont multipliées et certains journaux scientifiques sont presque entièrement consacrés à ce thème tels que "Regulated rivers : research and management» créé en 1986 ou "Rivers" en 1990. Avec la formalisation de I'IFIM (Instream Flow Incremental Methodology), dont le module d'évaluation de I'habitat PHABSIM (Physical HABitat SIMulation) devint rapidement 
un standard (REISER et al., 1989) pour les études d'impact des débits réservés, l'école de Fort Collins, USA (STALNAKER, 1979 ; BOVEE, 1982 : MILHOUS et al., 1989) a montré la voie du rapprochement entre sciences physiques et sciences biologiques. Un débat très riche s'en est suivi, qui a animé la littérature avec des critiques parfois sévères mais certainement salutaires (MATHUR et al., 1985 ; SHIRVELL, 1986) et des défenses plus positives (ORTH, 1987 ; NESTLER et al., 1989). Beaucoup, dont STALNAKER (1993), s'accordent désormais pour penser que les démarches couplant hydraulique et biologie doivent faire l'objet de plus de validations vis-à-vis des populations de poisson tout en prenant mieux en compte le fonctionnement écologique des cours d'eau.

Le débat n'est pas moins riche dans une autre communauté de scientifiques travaillant plus au niveau des peuplements de poisson, à des échelles plus larges (MATTHEWS et HEINS, 1987). La théorie des îles biogéographiques de MACARTHUR et WILSON (1967) s'appliquait-elle aux cours d'eau ? Pouvait-on choisir son camp entre les partisans d'une régulation biotique des communautés ou ceux d'une régulation par le physique (YANT et al., 1984 ; GROSSMAN et al., 1985) ? Ne devait-on pas être plus circonspect avec HUBBS (1987) qui pensait que l'influence respective des deux types de contrôle est fonction des types de cours d'eau ou avec ZALEWSKI et NAIMAN (1985) qui plaidaient pour une pondération longitudinale de ces contrôles. Enfin, la théorie du patch dynamic concept, transposée aux hydrosystèmes (TOWNSEND, 1989 ; STATZNER et al., 1994), portait-elle les germes d'une théorie unificatrice ? Les travaux sur l'écomorphologie des poissons (WINEMILLER, 1991) complétaient-ils ceux cités précédemment ? SOUTHWOOD (1977) avait déjà parfaitement défini l'habitat comme un cadre dans lequel l'évolution forge les caractéristiques des stratégies vitales des espèces.

Le rapprochement des problématiques scientifiques communes aux hydrauliciens et aux biologistes avait été discuté lors d'un groupe de travail tenu à Arles en 1991. II a été concrétisé dans le cadre d'un programme de recherche sur l'environnement lancé par le CNRS et cofinancé par l'Orstom, associant la plupart des équipes françaises affichées sur ces thèmes. Après trois années de travail, il a paru nécessaire de confronter les résultats obtenus avec ceux de nos collègues scientifiques étrangers au cours d'un symposium international sur le thème des relations entre le poisson et son habitat.

Selon une procédure classique, une double sélection des contributions a eu lieu. Un premier choix a permis de déterminer les travaux qui ont été présentés oralement en session (41 communications) ou sous forme d'affiches (56 communications). Chaque session a bénéficié d'une conférence introductive invitée. Toutes les communications reçues à temps ont ensuite été soumises à publication selon les règles de sélection en vigueur. Les 41 textes sélectionnés sont présentés dans ce numéro spécial.

\section{CONTENU DE CE NUMÉRO SPÉCIAL}

Après un texte de mise en situation de C. LÉVÊQUE intitulé "L'habitat, être au bon endroit au bon moment», trois chapitres organisent les différentes contributions.

Le premier chapitre est dévolu à l'habitat à une échelle régionale et à la diversité piscicole. Dans le texte introductif, WINEMILLER et TONN discutent respectivement des aspects structurels et fonctionnels de la diversité piscicole et des processus de colonisation et d'extinction des communautés. Les principaux enjeux sont de connaître les relations entre populations et communautés, et également leurs liens avec les structures et la dynamique des écosystèmes. Différents paramètres de repérage au sein de différents bassins hydrographiques français ont permis à CHANGEUX et PONT et al. de proposer des axes régionaux et longitudinaux de structuration des peuplements de poisson. Une mise en perspective historique de ces peuplements est proposée pour le bassin de la Seine (France) par BELLIARD et al.

A l'aide de données originales provenant d'Europe et d'Afrique de l'Ouest, HUGUENY et al. essayent de caractériser les évolutions à long terme des peuplements de poisson entre des tendances déterministes et stochastiques au sens de GROSSMAN et al. (1985). CARREL et al. se focalisent quant à eux sur l'évolution temporelle d'un peuplement 
fluvial (Rhône aval) en tentant de la relier à l'hydrologie. Enfin, abordant des aspects plus fonctionnels, PONT et al. font l'inventaire des principales stratégies démographiques des poissons de la faune française d'eau douce.

Pour progresser dans ces différents domaines, le travail des chercheurs pourra être facilité par une meilleure maîtrise et une meilleure architecture de leurs jeux de données (SANLAVILLE-BOISSON et al.) ou par des outils plus conviviaux d'acquisition des paramètres morphométriques (SAGNES).

Le second chapitre regroupe les articles traitant de l'habitat à une échelle locale, qui correspond à l'échelle de perception individuelle des poissons. Cela suppose d'abord que l'analyse de la dimension physique soit compatible avec cette échelle grâce à des adaptations des outils existants ou la proposition d'approches nouvelles (HÉROUIN et al.) concernant I'hydrologie, la géomorphologie (DELACOSTE et al.) ou l'hydraulique (LAMOUROUX). Les deux contributions des deux lecteurs invités rappellent la difficulté de trouver le bon compromis entre les caractéristiques de l'espace réellement perçu par le poisson et l'espace échantillonnable pratiquement (BAIN) sans négliger les propriétés de déplacement des poissons encore mal prises en compte (FAUSCH et al.). Six textes (HELAND et al., GAUDIN et HELAND, VIGNES et HELAND, SEMPESKI et GAUDIN, ROUSSEL et BARDONNET, HEGGENES et al.) analysent en profondeur l'écoéthologie des jeunes stades de Salmonidés dès l'émergence jusqu'à leur première année. BARAS et al. ont suivi quant à eux le microhabitat de jeunes cyprinidés, pour lesquels NICOLAS et PONT soulignent l'importance du maintien des annexes fluviales. Les variations temporelles de l'habitat induites par l'hydrologie naturelle (BEAUDOU et al.) ou reproduites artificiellement (DEBOWSKI et BEALL) peuvent bousculer les schémas d'utilisation standard de l'espace par les poissons. Enfin, trois articles proposent des solutions biologiques nouvelles pour enrichir les simulations d'habitat des cours d'eau par prise en compte de nouvelles espèces de poisson (SEMPESKI et GAUDIN pour l'ombre commun) ou des invertébrés (WASSON et al.) et le traitement d'espèces sympatriques pour la truite fario et la truite arc-en-ciel (BARAN et al.).

Le troisième chapitre a été intitulé "Transfert des concepts et des outils à la gestion". II rassemble tout d'abord deux articles rappelant la logique de couplage entre approches physiques et modèles biologiques et présente un outil en version française "EVHA» dédié à l'application, qui permet de simuler l'habitat du poisson sous un environnement convivial (GINOT) suivi d'un outil de recherche pour la description de la morphodynamique fluviale (LE COARER et DUMONT). Concernant les diverses facettes de la simulation des habitats selon la logique "microhabitat", les textes de ORTH, conférencier invité, et de POUILLY et SOUCHON tirent les leçons d'une dizaine d'années d'expérience d'application. L'attention est attirée sur les besoins d'une meilleure prise en compte générale de la dimension biologique dans la conduite des études et dans l'interprétation des résultats. Un point est également fait sur les différentes formes de validation envisageables et déjà partiellement réalisées pour ce type d'approche. CAPRA et al. montrent comment mieux intégrer la dimension temporelle, alors que DELACOSTE et al. se focalisent sur le stade reproduction de la truite fario. Enfin, JOHNSON et al. décrivent un cas complet d'application de la méthode des microhabitats en Grande-Bretagne. Quatre articles (BARNARD et WYATT, BARNARD et al., MILNER et al., BARAN et al.) présentent ensuite une autre logique d'approche de modèles globaux de biomasse de Salmonidés corrélée à différents paramètres du bassin versant et du cours d'eau. La dernière présentation de HUUSKO et YRJÄNÄ termine sur une note optimiste, en montrant que l'application de principes écologiques permet de restaurer des cours d'eau de façon satisfaisante.

\section{DÉROULEMENT DU SYMPOSIUM}

Le symposium s'est tenu à Lyon/Villeurbanne (France) du 6 au 8 décembre 1994. II était patronné par le CNRS (PIREN, programme environnement dénommé, depuis, Vie et Société) représenté par A. PAVÉ et le GIP Hydrosystèmes, groupement des organismes de recherche français. II a rassemblé 215 participants de 25 nations. L'Europe avec $80 \%$ des présents était bien représentée. Les 10 séances de communications orales ont été présidées successivement par D. PONT, B. JALABERT, Y. SOUCHON, M.B. BAIN, B. de MERONA, 
J. ALLARDI, K.O. WINEMILLER, B. STATZNER, D. ORTH et K.D. FAUSCH. DeuX séances ont été réservées pour les communications affichées. Les résumés de toutes les communications sont disponibles auprès des organisateurs du symposium.

La rencontre entre deux courants de la recherche ichtyologique, une interdisciplinarité naissante entre sciences physiques et biologiques, les comparaisons intercontinentales qui ont été permises, nous font considérer que ce symposium a rempli toutes ses attentes. II nous a également semblé que la gastronomie lyonnaise et la fête des lumières du 8 décembre n'avaient pas laissé nos hôtes insensibles.

\section{RECOMMANDATIONS}

Face à une demande croissante en matière de protection, restauration et valorisation des hydrosystèmes continentaux, on peut considérer qu'il existe des réponses scientifiques et techniques qu'il conviendrait maintenant de mieux mobiliser, par un meilleur transfert des concepts, par une meilleure formation des techniciens et des décideurs et par la mise à disposition d'outils d'aide à la décision. La solidité de l'ensemble de l'édifice repose sur une bonne intégration des différentes échelles de connaissance du biome au bassin, jusqu'au microhabitat. La caractérisation de situations de référence et la maîtrise partagée des réseaux de mesures sur le long terme sont les garants d'une production scientifique de qualité. La meilleure prise en compte des facteurs de l'environnement et l'apport des approches modélisatrices sont porteurs d'espoir : celui d'être à même de prévoir l'évolution des compartiments biologiques dans un environnement soumis aux impacts des activités humaines. L'ambition, qui n'apparaît plus démesurée, est de proposer des scénarios d'évolution des peuplements de poisson en jouant sur des facteurs de contrôle comme l'hydrologie et l'hétérogénéité de l'habitat. C'est ce qui intéresse également les gestionnaires soucieux d'identifier les causes sur lesquelles il est possible d'agir pour faire évoluer favorablement les communautés de poisson. Les démarches de modélisation sont par nature réductionnistes, aussi faut-il veiller à bien maintenir un équilibre entre approches physiciennes et biologiques. Dans ce dernier cas, les interrogations sur le comportement des poissons, sur leurs réactions possibles par rapport à certaines contraintes du milieu physique ou biologique, sur leurs possibilités de choix, voire d'apprentissage, doivent trouver toute leur place. C'est à ce prix que nous pourrons mieux comprendre les relations entre la biodiversité fonctionnelle et la diversité des écosystèmes.

\section{PROLONGEMENTS}

Beaucoup des thèmes abordés dans ce symposium s'inscrivent dans un courant de pensée et de recherche en devenir, qui a été rassemblé sous le vocable d'habitat et hydraulique puis d'écohydraulique. Un premier colloque a été consacré à ce thème en 1994 à Trondheim (Norvège) sous l'égide de l'AIRH (Association Internationale pour la Recherche Hydraulique). II sera suivi par un autre symposium en juin 1996 à Québec (Canada). Le présent symposium a permis la constitution d'un réseau européen (ECHYDNAH, ECological HYDrology and Network data to manage Aquatic Habitat) connecté avec la mouvance de FRIEND (Flow Regimes from International Experimental Network Data) du PHI (Programme Hydrologique International) de l'UNESCO, lors d'une réunion le 9 décembre 1994 à Villeurbanne (Animateurs P. BREIL, France et B. HIGLER, Pays-Bas). Depuis, s'est également créé un groupe international supra-européen dénommé "International Aquatic Modelling Group» lors d'une réunion à Vienne (Autriche) les 31 août et $1^{\text {er }}$ septembre 1995.

\section{REMERCIEMENTS}

Le parfait déroulement du symposium a été rendu possible grâce au travail de son comité d'organisation : J. ALLARDI, M.F. BOSSENIE, P. GAUDIN (Président), M. HELAND, B. HUGUENY, B. de MERONA, E. PATTEE, D. PONT, Y. SOUCHON (Vice-président) et E. VIGNEUX, et à la compétence et au dévouement de son équipe technique : MarieFrançoise ARENS, Denise BICHET, Anne EICHOLTZ, Denise GROLET, Nadjette HOURIEZ, Nathalie LYVET et Fleur TARI, assistées de doctorants des laboratoires 
d'Hydroécologie Quantitative du CEMAGREF et de l'équipe d'Ecologie Fonctionnelle du Laboratoire d'Ecologie des Eaux Douces et des Grands Fleuves.

L'élaboration du programme scientifique et la sélection des articles pour ce numéro spécial ont été assurées par les membres du comité scientifique : J. ALLARDI, J.L. BAGLINIĖRE, M.B. BAIN, P. BERREBI, J. BLONDEL, P. BOET, I. COWX, K.D. FAUSCH, R. GALZIN, P. GAUDIN, C. GRANADO-LORENCIO, J. HEGGENES, M. HELAND, B. HUGUENY, P. KEITH, C. LÉVÊQUE (Président), B. de MERONA, D.J. ORTH, E. PATTEE, D. PONT, C. SABATON, Y. SOUCHON, B. STATZNER, W.M. TONN, E. VIGNEUX, R. WELCOMME, K.O. WINEMILLER, assistés d'experts anonymes.

Merci à E. VIGNEUX, rédacteur en chef du Bulletin Français de la Pêche et de la Pisciculture, d'avoir accepté de nous ouvrir la revue dont il coordonne l'édition et à D.J. ORTH d'avoir mis ses compétences à notre service pour superviser l'édition des textes en langue anglaise.

Merci enfin à E. PATTEE pour son efficacité désormais légendaire qui s'est manifestée à chacune des étapes de ce symposium.

\section{Le symposium a été organisé avec la participation financière et matérielle de :}

- Agence de l'Eau Rhône-Méditerranée-Corse,

- Centre National du Machinisme Agricole, des Eaux et des Forêts (CEMAGREF),

- Compagnie Nationale du Rhône (CNR),

- Centre National de la Recherche Scientifique (CNRS),

- Conseil Régional Rhône-Alpes,

- Conseil Supérieur de la Pêche (CSP),

- Electricité de France (EDF : Direction Etudes et Recherches, et Direction Production Transport),

- Institut Français de Recherche pour l'Exploitation de la Mer (IFREMER),

- Institut Français pour le Développement en Coopération (ORSTOM),

- Institut National de la Recherche Agronomique (INRA),

- Ministère de l'Environnement (Direction de l'eau),

- Mairie de Villeurbanne,

- Relations Internationales, Université Lyon I.

\section{BIBLIOGRAPHIE}

BOVEE K.D., 1982. A guide to stream habitat analysis using the Instream Flow Incremental Methodology. U.S.D.I. Fish and Wildlife Service, Office of Biological Services, Fort Collins, Colorado, Instream Flow Information Paper $n^{\circ} 12$, FWS/OBS 82/86, 248 p.

GROSSMAN G.D., FREEMAN M.C., MOYLE P.B., WHITAKER J.O., 1985. Stochasticity and assemblage organization in an Indiana stream fish assemblage. The American Naturalist, 126, 275-285.

HUBBS C., 1987. Summary of the symposium. In : MATTHEWS W.J. \& HEINS D.C. (eds), Community and evolutionary ecology of North American stream fishes, University of Oklahoma press, 265-267.

LÉGER L., 1909. Principe de la méthode rationnelle du peuplement des cours d'eau à salmonidés. Travaux du Laboratoire de Pisciculture de Grenoble, 1, 533-568.

MACARTHUR R.H., WILSON E.O., 1967. The theory of island biogeography. Princeton University press, 1, Princeton, New Jersey.

MATHUR D., BASON W.H., PURDY E.J., SILVER C.A., 1985. A critique of the Instream Flow Incremental Methodology. Canadian Journal of Fisheries and Aquatic Sciences, $42,825-831$.

MATTHEWS W.J., HEINS D.C., 1987. Community and Evolutionary Ecology of North American Stream Fishes, Norman, University of Oklahoma Press, $310 \mathrm{p}$. 
MILHOUS R.T., UPDIKE M.A., SCHNEIDER D.M., 1989. Physical habitat simulation system reference manual - version II. Fish and Wildlife Service, Office of Biological Service, Ft Collins, Biological Report 89 (16), 26 FWS/OBS 89/16, 216 p.

NESTLER J.M., MILHOUS R.T., LAYZER J.B., 1989. Instream habitat modeling techniques. In : GORE J.A. \& PETTS G.E. (eds), Alternatives in regulated river management, CRC Press, Baca Raton, Florida, 295-315.

ORTH D.J., 1987. Ecological considerations in the development and application of instream flow habitat models. Regulated Rivers : Research \& Management, 1, 171-181.

REISER D.W., WESCHE T.A., ESTES C., 1989. Status of instream flow legislation and practices in North American. Fisheries, 14, 22-29.

SHIRVELL C.S., 1986. Pitfalls of physical habitat simulation in the Instream Flow Incremental Methodology. Canadian Technical Report of Fisheries and Aquatic Sciences, $1460,68 \mathrm{p}$.

SOUTHWOOD T.R.E., 1977. Habitat : the templet for ecological strategies. Journal of Animal Ecology, 46, 337-367.

STALNAKER C.B., 1979. The use of habitat structure preferenda for establishing flow regimes necessary for maintenance of fish habitat. In : WARD J.V. \& STANFORD J.A. (eds), The ecology of regulated streams, New York, 326-337.

STALNAKER C.B., 1993. Fish habitat evaluation models in environmental assessments. In : HILDEBRAND S.G. \& CANNON J.B. (eds), Environmental analysis. The NEPA experience. Lewis, Boca Raton, Florida, 140-162.

STATZNER B., RESH V.H., ROUX A.L., 1994. The synthesis of long-term ecological research in the context of concurrently developed ecological theory : design of a research strategy for the Upper Rhône River and its floodplain. Freshwater Biology, 31, 253-263.

TOWNSEND C.R., 1989. The patch dynamic concept of stream community ecology. Journal of North American Benthological Society, 8, 36-50.

WATERS B.F., 1976. A methodology for evaluating the effects of different streamflows on salmonid habitat. In : ORSBORN J.F. \& ALLMAN C.H. (eds), Instream Flow Needs, American Fisheries Society, Western Division, Bethesda, Maryland, 254-266.

WINEMILLER K.O., 1991. Ecomorphological diversification of freshwater fish assemblages from five biotic regions. Ecol. Monogr., 61, 343-365.

YANT P.R., KARR J.R., ANGERMEIER P.L., 1984. Stochasticity in stream fish communities : an alternative interpretation. The American Naturalist, 124, 573-582.

ZALEWSKI M., NAIMAN R.J., 1985. The regulation of riverine fish communities by a continuum of abiotic-biotic factors. In : ALABASTER J.S. (ed.), Habitat modification and freshwater fisheries, Butterworth Scientific, London, 3-9. 